\title{
The Hopf Bifurcation for a Predator-Prey System with $\theta$-Logistic Growth and Prey Refuge
}

\author{
Shaoli Wang and Zhihao Ge \\ School of Mathematics and Information Sciences, Henan University, Kaifeng 475001, China \\ Correspondence should be addressed to Shaoli Wang; wangshaoli110@163.com
}

Received 12 April 2013; Accepted 6 June 2013

Academic Editor: Luca Guerrini

Copyright (C) 2013 S. Wang and Z. Ge. This is an open access article distributed under the Creative Commons Attribution License, which permits unrestricted use, distribution, and reproduction in any medium, provided the original work is properly cited.

\begin{abstract}
The Hopf bifurcation for a predator-prey system with $\theta$-logistic growth and prey refuge is studied. It is shown that the ODEs undergo a Hopf bifurcation at the positive equilibrium when the prey refuge rate or the index $\theta$ passed through some critical values. Time delay could be considered as a bifurcation parameter for DDEs, and using the normal form theory and the center manifold reduction, explicit formulae are derived to determine the direction of bifurcations and the stability and other properties of bifurcating periodic solutions. Numerical simulations are carried out to illustrate the main results.
\end{abstract}

\section{Introduction}

The construction and study of models for the population dynamics of predator-prey systems have long been and will continue to be one of the dominant themes in both ecology and mathematical ecology since the famous Lotka-Volterra equations. In recent years, the study of the consequences of hiding behavior of prey on the dynamics of predatorprey interactions has been an active topic [1-5]. Some of the empirical and theoretical work have investigated the effects of prey refuges and drawn a conclusion that the refuges used by prey have a stabilizing effect on the considered interactions and prey extinction can be prevented by the addition of refuges [6-12].

Motivated by the work of Ko and Ryu [13] and Tsoularis and Wallace [14], we construct the following $\theta$-logistic growth predator-prey system with Holling type-II functional response and prey refuge:

$$
\begin{gathered}
\dot{x}=r x\left[1-\left(\frac{x}{K}\right)^{\theta}\right]-\frac{\beta \varepsilon x y}{1+\varepsilon x}, \\
\dot{y}=\frac{\beta \varepsilon x y}{1+\varepsilon x}-a y,
\end{gathered}
$$

where $x$ and $y$ represent the densities of prey and predator, respectively, and $r, K, \theta, \beta, \varepsilon$, and $a$ are all positive constants and have their biological meanings accordingly. $r$ is the $\theta$ logistic intrinsic growth rate of the prey in the absence of the predator; $K$ is the carrying capacity; $\theta$ is the logistic index; $\beta$ is the predation rate of predator; $0<\varepsilon<1$ and $1-\varepsilon$ is the prey refuge rate; $a$ is the death rate of the predator.

By assuming that the reproduction of predator after predating the prey will not be instantaneous but mediated by some discrete time lag required for gestation of predator, we incorporate a delay in system (1) to make the model more realistic. We aim to discuss the effect of time delay due to gestation of the predator on the global dynamics of system (1). To this end, we consider the following delayed predatorprey system with $\theta$-logistic growth and prey refuge:

$$
\begin{gathered}
\dot{x}=r x\left[1-\left(\frac{x}{K}\right)^{\theta}\right]-\frac{\beta \varepsilon x y}{1+\varepsilon x}, \\
\dot{y}=\frac{e^{-m \tau} \beta \varepsilon x(t-\tau) y(t-\tau)}{1+\varepsilon x(t-\tau)}-a y,
\end{gathered}
$$

where $m$ is positive constant. The constant $\tau$ denotes a time delay due to the gestation of the predator and the term $e^{-m \tau}$ denotes the probability of the predators, which capture the prey at time $t-\tau$ and still alive at times $t$. 
The initial conditions for system (2) take the form

$$
\begin{gathered}
x(\eta)=\phi(\eta) \geq 0, \quad y(\eta)=\psi(\eta) \geq 0, \\
\eta \in[-\tau, 0], \quad \phi(0)>0, \quad \psi(0)>0,
\end{gathered}
$$

where $(\phi(\eta), \psi(\eta)) \in C\left([-\tau, 0], R_{+0}^{2}\right)$, the Banach space of continuous functions mapping the interval $[-\tau, 0]$ into $R_{+0}^{2}$, where $R_{+0}^{2}=\{(x, y): x \geq 0, y \geq 0\}$.

It is well known by the fundamental theory of functional differential equations [15] that system (2) has a unique solution $x(t)$ and $y(t)$ satisfying initial conditions (3).

The organization of this paper is as follows. In Section 2, we show the positivity and the boundedness of solutions of system (2) with initial conditions (3). In Section 3, we discuss the stability of boundary equilibria of system (2). In Section 4, we study the existence of Hopf bifurcations for system (1) and (2) at the positive equilibrium. In Section 5, using the normal form theory and the center manifold reduction, explicit formulae are derived to determine the direction of bifurcations and the stability and other properties of bifurcating periodic solutions. Finally, numerical simulations are carried out to illustrate the main results, and a brief discussion is given to conclude this work in Section 6.

\section{Positivity and Boundedness}

In this section, we show the positivity and boundedness of solutions of system (2) with initial conditions (3).

\subsection{Positivity of Solutions}

Theorem 1. Solutions of system (2) with initial conditions (3) are positive for all $t \geq 0$.

Proof. Let $(x(t), y(t))$ be a solution of system (2) with initial conditions (3). From the first equation of system (2), we have

$$
x(t)=x(0) e^{\int_{0}^{t}\left(r\left(1-(x(\varsigma) / K)^{\theta}\right)-\beta \varepsilon y(\varsigma) /(1+\varepsilon x(\varsigma))\right) d \varsigma} .
$$

Since $\phi(\eta) \geq 0$. Hence, $x(t)$ is positive.

To show that $y(t)$ is positive on $[0,+\infty)$, suppose that there exists $t_{1}$ such that $y\left(t_{1}\right)=0$, and $y(t)>0$ for $t \in\left[0, t_{1}\right)$. Then $\dot{y}\left(t_{1}\right) \leq 0$. From the second equation of (2), we have

$$
\begin{aligned}
\dot{y}\left(t_{1}\right) & =\frac{e^{-m \tau} \beta \varepsilon x\left(t_{1}-\tau\right) y\left(t_{1}-\tau\right)}{1+\varepsilon x\left(t_{1}-\tau\right)}-a y\left(t_{1}\right) \\
& =\frac{e^{-m \tau} \beta \varepsilon x\left(t_{1}-\tau\right) y\left(t_{1}-\tau\right)}{1+\varepsilon x\left(t_{1}-\tau\right)} \\
& >0
\end{aligned}
$$

which is a contradiction.

Next, we will prove the boundedness of solutions.

\subsection{Boundedness of Solutions}

Theorem 2. Positive solutions of system (2) with initial conditions (3) are ultimately bounded.
Proof. Let $(x(t), y(t))$ be a solution of system (2) with initial conditions (3). From the first equation of (2), we have

$$
\dot{x} \leq r x\left[1-\left(\frac{x}{K}\right)^{\theta}\right],
$$

which yields

$$
x(t) \leq \frac{x_{0} K}{\left[x_{0}^{\beta}+\left(K^{\beta}-x_{0}^{\beta}\right) e^{-r \theta t}\right]^{1 / \theta}},
$$

and therefore

$$
\limsup _{t \rightarrow+\infty} x(t) \leq K
$$

Hence, for $\rho$ sufficiently small, there is a $T_{1}>0$ such that if $t>T_{1}, x(t)<K+\rho$. Set

$$
V(t)=x(t-\tau) e^{-m \tau}+y(t) .
$$

Calculating the derivative of $V$ along solutions of system (2), we obtain

$$
\begin{aligned}
\dot{V}(t) & =e^{-m \tau} r x(t-\tau)\left[1-\left(\frac{x(t-\tau)}{K}\right)^{\theta}\right]-a y(t) \\
& \leq e^{-m \tau} r x(t-\tau)-a y(t) \\
& \leq M_{0}-a V(t),
\end{aligned}
$$

where $M_{0}=e^{-m \tau} r(K+\rho)$. Then there exists an $M>0$, depending only on the parameters of system (2), such that $V(t) \leq M$ for all $t$ large enough. Then $x(t), y(t)$ have an ultimately above bound.

\section{Stability of the Boundary Equilibria}

In this section, we discuss the stability of the boundary equilibria of system (2) with initial conditions (3).

We denote $R_{0}=K \varepsilon\left(\beta e^{-m \tau}-a\right) / a$ and always assume $\beta>a$. The system (2) always has two boundary equilibria $E_{0}=(0,0)$ and $E_{1}=(K, 0)$; if $R_{0}>1$, it also has a positive equilibrium $E^{*}=\left(x^{*}, y^{*}\right)$, where

$$
\begin{gathered}
x^{*}=\frac{a}{\varepsilon\left(\beta e^{-m \tau}-a\right)}, \\
y^{*}=\frac{r\left[1-\left(x^{*} / K\right)^{\theta}\right]\left(1+\varepsilon x^{*}\right)}{\beta \varepsilon} .
\end{gathered}
$$

Now we consider the stability of boundary equilibria. For $E_{0}=(0,0)$, the corresponding characteristic equation is

$$
(\lambda-r)(\lambda+a)=0
$$

and the roots are

$$
\lambda_{1}=r>0, \quad \lambda_{2}=-a<0,
$$

which implies that the equilibrium $E_{0}$ is always unstable. 

tion is

For $E_{1}=(K, 0)$, the corresponding characteristic equa-

$$
(\lambda+r \theta)\left(\lambda+a-\frac{K \beta \varepsilon e^{-m \tau}}{1+K \varepsilon e^{-m \tau}} e^{-\lambda \tau}\right)=0
$$

It follows that

$$
\lambda_{1}=-r \theta
$$

or

$$
\lambda+a-\frac{K \beta \varepsilon e^{-m \tau}}{1+K \varepsilon} e^{-\lambda \tau}=0 .
$$

Denote

$$
f(\lambda)=\lambda+a-\frac{K \beta \varepsilon e^{-m \tau}}{1+K \varepsilon} e^{-\lambda \tau}
$$

and then we have

$$
\begin{aligned}
f(0) & =a-\frac{K \beta \varepsilon e^{-m \tau}}{1+K \varepsilon} \\
& =\frac{1}{1+K \varepsilon}\left(a+K a \varepsilon-K \beta \varepsilon e^{-m \tau}\right) \\
& =\frac{a}{1+K \varepsilon}\left(1-R_{0}\right), \\
\lim _{\lambda \rightarrow \infty} f(\lambda) & =\infty
\end{aligned}
$$

for any $\tau \geq 0$. Hence $f(\lambda)=0$ has no positive root for $R_{0} \leq 1$, and at least one positive for $R_{0}>1$. Therefore, for all $\tau \geq 0$, the equilibrium $E_{1}$ is stable when $R_{0} \leq 1$ and unstable when $R_{0}>1$.

Summarizing the discussion above, we obtain the following conclusion.

Theorem 3. (i) The equilibrium $E_{0}$ is always unstable for all $\tau \geq 0$.

(ii) The equilibrium $E_{1}$ is stable when $R_{0} \leq 1$ and unstable when $R_{0}>1$ for all $\tau \geq 0$.

\section{The Hopf Bifurcation}

4.1. The Hopf Bifurcation of ODEs. When $R_{0}>1$, the system (2) also has a positive equilibrium $E^{*}$. The characteristic of the linearized system of (2) near the infected equilibrium $E^{*}$ is given by

$$
P(\lambda, \tau)+Q(\lambda, \tau) e^{-\lambda \tau}=0
$$

where

$$
\begin{aligned}
P(\lambda, \tau)= & \lambda^{2}+b_{1}(\tau) \lambda+b_{2}(\tau), \\
Q(\lambda, \tau)= & b_{3}(\tau) \lambda+b_{4}(\tau), \\
b_{1}(\tau)= & a+r \theta\left(\frac{x^{*}}{K}\right)^{\theta}-\frac{\beta \varepsilon^{2} x^{*} y^{*}}{\left(1+\varepsilon x^{*}\right)^{2}}, \\
b_{2}(\tau)= & a\left[r \theta\left(\frac{x^{*}}{K}\right)^{\theta}-\frac{\beta \varepsilon^{2} x^{*} y^{*}}{\left(1+\varepsilon x^{*}\right)^{2}}\right], \\
b_{3}(\tau)= & -\frac{\beta \varepsilon x^{*} e^{-m \tau}}{1+\varepsilon x^{*}}, \\
b_{4}(\tau)= & \frac{\beta^{2} \varepsilon^{2} x^{*} y^{*} e^{-m \tau}}{\left(1+\varepsilon x^{*}\right)^{3}} \\
& -\frac{\beta \varepsilon x^{*} e^{-m \tau}}{1+\varepsilon x^{*}}\left[r \theta\left(\frac{x^{*}}{K}\right)^{\theta}-\frac{\beta \varepsilon^{2} x^{*} y^{*}}{\left(1+\varepsilon x^{*}\right)^{2}}\right] .
\end{aligned}
$$

When $\tau=0, R_{0}$ becomes $R_{0}^{*}=K \varepsilon(\beta-a) / a$ and (19) becomes

$$
\lambda^{2}+a_{1}(0) \lambda+a_{2}(0)=0
$$

where

$$
\begin{aligned}
a_{1}(0)= & b_{1}(0)+b_{3}(0) \\
= & a+r \theta\left(\frac{x^{*}}{K}\right)^{\theta}-\frac{\beta \varepsilon^{2} x^{*} y^{*}}{\left(1+\varepsilon x^{*}\right)^{2}}-\frac{\beta \varepsilon x^{*}}{1+\varepsilon x^{*}} \\
= & r \theta\left(\frac{x^{*}}{K}\right)^{\theta}-\frac{a \varepsilon y^{*}}{1+\varepsilon x^{*}} \\
= & r\left(\theta+\frac{a}{\beta}\right)\left(\frac{a}{K \varepsilon(\beta-a)}\right)^{\theta}-\frac{a r}{\beta}, \\
a_{2}(0)= & b_{2}(0)+b_{4}(0) \\
= & a\left[r \theta\left(\frac{x^{*}}{K}\right)^{\theta}-\frac{\beta \varepsilon^{2} x^{*} y^{*}}{\left(1+\varepsilon x^{*}\right)^{2}}\right]+\frac{\beta^{2} \varepsilon^{2} x^{*} y^{*}}{\left(1+\varepsilon x^{*}\right)^{3}} \\
& -\frac{\beta \varepsilon x^{*}}{1+\varepsilon x^{*}}\left[r \theta\left(\frac{x^{*}}{K}\right)^{\theta}-\frac{\beta \varepsilon^{2} x^{*} y^{*}}{\left(1+\varepsilon x^{*}\right)^{2}}\right] \\
= & \frac{\beta^{2} \varepsilon^{2} x^{*} y^{*}}{\left(1+\varepsilon x^{*}\right)^{3}}>0 .
\end{aligned}
$$

Theorem 4. (i) If $R_{0}^{*}>1$ and $a_{1}(0)>0$, then the positive equilibrium $E^{*}$ of system (1) is asymptotically stable.

(ii) If $R_{0}^{*}>1$ and $a_{1}(0)<0$, then system (1) is unstable.

(iii) Suppose that $R_{0}^{*}>1$. Then system (1) undergoes a Hopf bifurcation when $\varepsilon(\operatorname{or} \theta)$ passes through $\varepsilon_{c}\left(\operatorname{or} \theta_{c}\right)$.

Proof. Obviously (i) and (ii) hold. 
(iii) From (23), we know that the root of the characteristic equation (22) satisfies

$$
\begin{aligned}
\operatorname{Re} \lambda & =-\frac{b_{1}(0)+b_{3}(0)}{2} \\
& =\frac{r}{2}\left[\frac{a}{\beta}-(\beta+\theta)\left(\frac{a}{K \varepsilon(\beta-a)}\right)^{\theta}\right] .
\end{aligned}
$$

Solving $\operatorname{Re} \lambda=0$, we have

$$
\varepsilon_{c}=\frac{a(1+\beta \theta / a)^{1 / \theta}}{K(\beta-a)}
$$

or

$$
a=\left(a+\beta \theta_{c}\right)\left[\frac{a}{K \varepsilon(\beta-a)}\right]^{\theta_{c}} .
$$

Calculating the derivative, we obtain

$$
\left.\frac{d \operatorname{Re}(\lambda(\varepsilon))}{d \varepsilon}\right|_{\varepsilon=\varepsilon_{c}}=-\left.\frac{\operatorname{ar} \theta(a+\beta \theta)}{2 K \beta(\beta-a)}\left[\frac{a}{K \varepsilon(\beta-a)}\right]^{\theta-1}\right|_{\varepsilon=\varepsilon_{c}}<0
$$

or

$$
\begin{aligned}
\left.\frac{d \operatorname{Re}(\lambda(\theta))}{d \theta}\right|_{\theta=\theta_{c}}= & -\frac{r}{2}\left[\frac{a}{K \varepsilon(\beta-a)}\right]^{\theta} \\
& -\left.\frac{r \theta(a+\beta \theta)}{2 \beta}\left[\frac{a}{K \varepsilon(\beta-a)}\right]^{\theta-1}\right|_{\theta=\theta_{c}}<0 .
\end{aligned}
$$

Hence the positive equilibrium is stable when $\varepsilon<\varepsilon_{c}\left(\theta<\theta_{c}\right)$ and unstable when $\varepsilon>\varepsilon_{c}\left(\theta>\theta_{c}\right)$. Thus Hopf bifurcation occurs at $\varepsilon=\varepsilon_{c}\left(\theta=\theta_{c}\right)$.

Example 5. We choose a series of parameter $r=0.1, K=10$, $\beta=0.2, a=0.13$, and $\theta=3$, then $\varepsilon_{c} \approx 0.33$. If $\varepsilon=0.3<\varepsilon_{c}$, then $R_{0}^{*} \approx 1.62>1$ and $a_{1}(0) \approx 0.022>0$, thus the system (1) is stable (see Figure $1(\mathrm{a})$ ); if $\varepsilon=0.42>\varepsilon_{c}$, then $R_{0}^{*} \approx 2.26>1$ and $a_{1}(0) \approx-0.033<0$, and thus the system (1) is unstable (see Figure 1(b)).

If we choose a series of parameter $r=0.1, K=10, \beta=$ $0.2, a=0.13$, and $\varepsilon=0.3$, then $R_{0}^{*} \approx 1.62>1$ and $\theta_{c} \approx 4.18$ $\left(\theta_{c} \approx 2.08 \times 10^{-22}\right.$ is unserviceable). If $\theta=4<\theta_{c}$, then $a_{1}(0) \approx$ $0.003>0$, and thus the system (1) is stable (see Figure $2(\mathrm{a})$ ); if $\theta=5>\theta_{c}$, then $a_{1}(0) \approx-0.014<0$, and thus the system (1) is unstable (see Figure 2(b)).

4.2. The Hopf Bifurcation of DDEs. In the following, we investigate the existence of purely imaginary roots $\lambda=$ $i \omega(\omega>0)$ to (19). Equation (19) takes the form of a second-degree exponential polynomial in $\lambda$, with all the coefficients of $P$ and $Q$ depending on $\tau$. Beretta and Kuang [16] established a geometrical criterion which gives the existence of purely imaginary root of a characteristic equation with delay dependent coefficients.
In order to apply the criterion due to Beretta and Kuang [16], we need to verify the following properties for all $\tau \in$ $\left[0, \tau_{\max }\right)$, where $\tau_{\max }$ is the maximum value in which $E^{*}$ exists.

(a) $P(0, \tau)+Q(0, \tau) \neq 0$;

(b) $P(i \omega, \tau)+Q(i \omega, \tau) \neq 0$;

(c) $\lim \sup \{|P(\lambda, \tau) / Q(\lambda, \tau)|:|\lambda| \rightarrow \infty, \operatorname{Re} \lambda \geq 0\}<1$;

(d) $F(\omega, \tau)=|P(i \omega, \tau)|^{2}-|Q(i \omega, \tau)|^{2}$ has a finite number of zeros;

(e) each positive root $\omega(\tau)$ of $F(\omega, \tau)=0$ is continuous and differentiable in $\tau$ whenever it exists.

Here, $P(\lambda, \tau)$ and $Q(\lambda, \tau)$ are defined as in (20).

Let $\tau \in\left[0, \tau_{\max }\right)$, and using (20) and (21), we have

$$
P(0, \tau)+Q(0, \tau)=b_{2}(\tau)+b_{4}(\tau)=\frac{\beta^{2} \varepsilon^{2} x^{*} y^{*} e^{-m \tau}}{\left(1+\varepsilon x^{*}\right)^{3}}>0,
$$

and then

$$
\begin{aligned}
& P(i \omega, \tau)+Q(i \omega, \tau) \\
& =-\omega^{2}+b_{2}(\tau)+b_{4}(\tau)+i \omega\left[b_{1}(\tau)+b_{3}(\tau)\right] \neq 0 .
\end{aligned}
$$

Therefore, (a) and (b) are satisfied.

From (20), we know that

$$
\begin{aligned}
\lim _{|\lambda| \rightarrow+\infty}\left|\frac{Q(\lambda, \tau)}{P(\lambda, \tau)}\right| & =\lim _{|\lambda| \rightarrow+\infty}\left|\frac{b_{3}(\tau) \lambda+b_{4}(\tau)}{\lambda^{2}+b_{1}(\tau) \lambda+b_{2}(\tau)}\right| \\
& =0 .
\end{aligned}
$$

Therefore, (c) follows.

Let $F$ be defined as in (d). From

$$
\begin{gathered}
|P(i \omega, \tau)|^{2}=\omega^{4}+\left[b_{1}(\tau)^{2}-2 b_{2}(\tau)\right] \omega^{2}+b_{2}(\tau)^{2}, \\
|Q(i \omega, \tau)|^{2}=b_{3}(\tau)^{2} \omega^{2}+b_{4}(\tau)^{2}
\end{gathered}
$$

we have

$$
F(\omega, \tau)=\omega^{4}+a_{1}(\tau) \omega^{2}+a_{2}(\tau)
$$

where

$$
\begin{gathered}
a_{1}(\tau)=b_{1}^{2}(\tau)-2 b_{2}(\tau)-b_{3}^{2}(\tau), \\
a_{2}(\tau)=b_{2}^{2}(\tau)-b_{4}^{2}(\tau) .
\end{gathered}
$$

It is obvious that property $(\mathrm{d})$ is satisfied. Let $\left(\omega_{0}, \tau_{0}\right)$ be a point of its domain of definition such that $F\left(\omega_{0}, \tau_{0}\right)=$ 0 . We know that the partial derivatives $F_{\omega}$ and $F_{\tau}$ exist and are continuous in a certain neighborhood of $\left(\omega_{0}, \tau_{0}\right)$, and $F_{\omega}\left(\omega_{0}, \tau_{0}\right) \neq 0$. By implicit function theorem, (e) is also satisfied.

Now let $\lambda=i \omega(\omega>0)$ be a root of (19). Substituting it into (19) and separating the real and imaginary parts yields

$$
\begin{gathered}
\omega^{2}-b_{2}(\tau)=b_{3}(\tau) \omega \sin \omega \tau+b_{4}(\tau) \cos \omega \tau, \\
b_{1}(\tau) \omega=b_{4}(\tau) \sin \omega \tau-b_{3}(\tau) \omega \cos \omega \tau .
\end{gathered}
$$



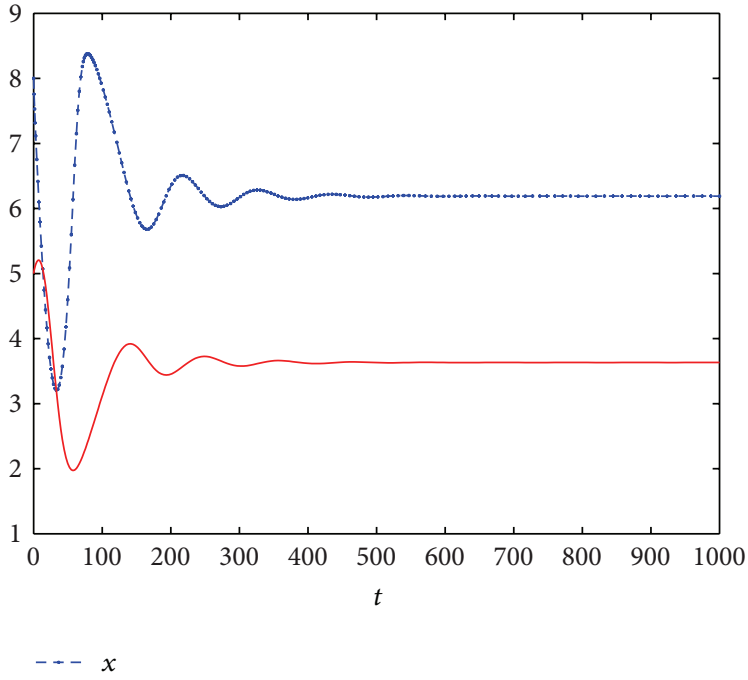
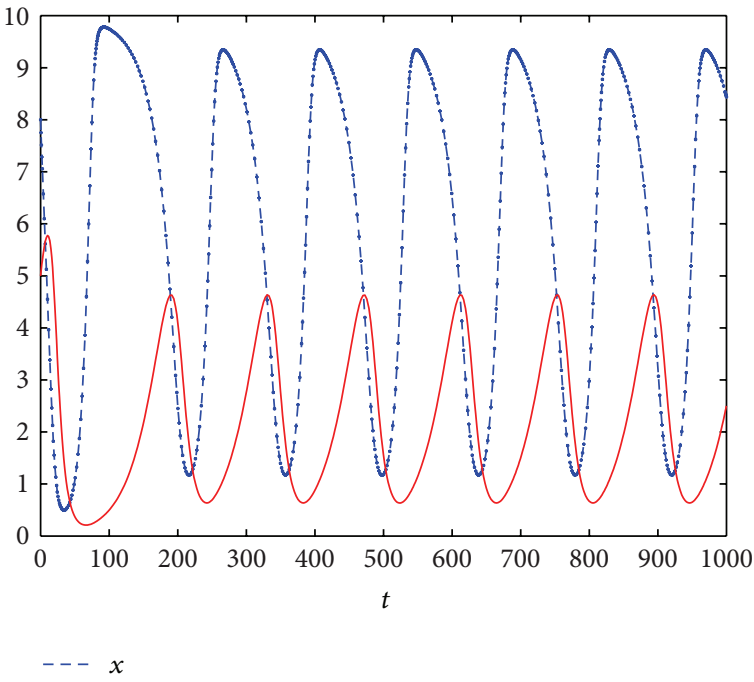

$-y$

(a)

(b)

FIGURE 1: The infected steady state $E^{*}$ of system (1) is asymptotically stable when $\varepsilon=0.3$ (a) and unstable when $\varepsilon=0.42$ (b). The rest parameter values are $r=0.1, K=10, \beta=0.2, a=0.13$, and $\theta=3$, and the initial values are $x_{0}=8$ and $y_{0}=5$.
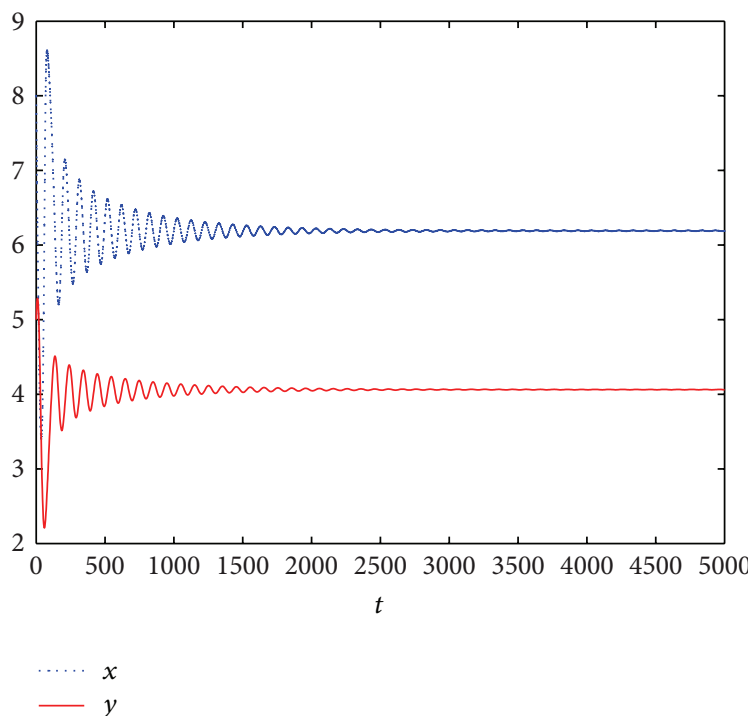

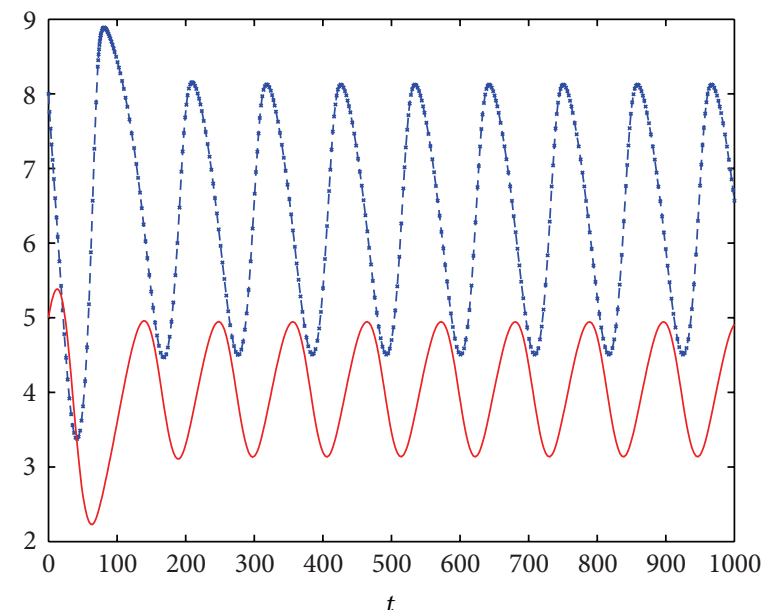

$-\cdots x$

(a)

(b)

Figure 2: The infected steady state $E^{*}$ of system (1) is asymptotically stable when $\theta=4$ (a), and unstable when $\theta=5$ (b). The rest parameter values are $r=0.1, K=10, \beta=0.2, a=0.13$, and $\varepsilon=0.3$, and the initial values are $x_{0}=8$ and $y_{0}=5$.

From (36), it follows that

$$
\begin{aligned}
\sin \omega \tau & =\frac{\omega\left[b_{3}(\tau)\left(\omega^{2}-b_{2}(\tau)\right)+b_{1}(\tau) b_{4}(\tau)\right]}{b_{3}(\tau) \omega^{2}+b_{4}^{2}(\tau)}, \\
\cos \omega \tau & =\frac{\left(b_{4}(\tau)-b_{1}(\tau) b_{3}(\tau)\right) \omega^{2}-b_{2}(\tau) b_{4}(\tau)}{b_{3}(\tau) \omega^{2}+b_{4}^{2}(\tau)} .
\end{aligned}
$$

By the definitions of $P(\lambda, \tau), Q(\lambda, \tau)$ as in (20), and applying the property (a), (20) can be written as

$$
\begin{aligned}
\sin \omega \tau & =\operatorname{Im} \frac{P(i \omega, \tau)}{Q(i \omega, \tau)} \\
\cos \omega \tau & =-\operatorname{Re} \frac{P(i \omega, \tau)}{Q(i \omega, \tau)},
\end{aligned}
$$


which yields

$$
|P(i \omega, \tau)|^{2}=|Q(i \omega, \tau)|^{2} .
$$

Assume that $I \in R_{+0}$ is the set where $\omega(\tau)$ is a positive root of

$$
F(\omega, \tau)=|P(i \omega, \tau)|^{2}-|Q(i \omega, \tau)|^{2}
$$

and for $\tau \notin I, \omega(\tau)$ is not defined. Then for all $\tau$ in $I, \omega(\tau)$ is satisfying

$$
F(\omega, \tau)=0
$$

Let $\omega^{2}=h$; we have that

$$
F(h, \tau)=h^{2}+a_{1}(\tau) h+a_{2}(\tau)=0
$$

We set

$$
\begin{aligned}
\Delta(\tau) & =a_{1}(\tau)^{2}-4 a_{2}(\tau) \\
& =\left[b_{1}^{2}(\tau)-2 b_{2}(\tau)-b_{3}^{2}(\tau)\right]^{2}-4\left[b_{2}^{2}(\tau)-b_{4}^{2}(\tau)\right] .
\end{aligned}
$$

Then, when $\Delta(\tau) \geq 0, F(h, \tau)=0$ has real roots given by

$$
\begin{aligned}
& h_{+}(\tau)=\frac{-a_{1}(\tau)+\sqrt{\Delta(\tau)}}{2}, \\
& h_{-}(\tau)=\frac{-a_{1}(\tau)-\sqrt{\Delta(\tau)}}{2} .
\end{aligned}
$$

Note that

$$
\begin{aligned}
a_{1}(\tau)= & b_{1}^{2}(\tau)-2 b_{2}(\tau)-b_{3}^{2}(\tau) \\
= & {\left[a+r \theta\left(\frac{x^{*}}{K}\right)^{\theta}-\frac{\beta \varepsilon^{2} x^{*} y^{*}}{\left(1+\varepsilon x^{*}\right)^{2}}\right]^{2} } \\
& -2 a\left[r \theta\left(\frac{x^{*}}{K}\right)^{\theta}-\frac{\beta \varepsilon^{2} x^{*} y^{*}}{\left(1+\varepsilon x^{*}\right)^{2}}\right]-\left(\frac{\beta \varepsilon x^{*} e^{-m \tau}}{1+\varepsilon x^{*}}\right)^{2} \\
= & {\left[r \theta\left(\frac{x^{*}}{K}\right)^{\theta}-\frac{\beta \varepsilon^{2} x^{*} y^{*}}{\left(1+\varepsilon x^{*}\right)^{2}}\right]^{2}>0, }
\end{aligned}
$$

and summarizing the discussion above, we have the following conclusion.

Proposition 6. If $\Delta(\tau) \geq 0$ and $a_{2}(\tau)<0$, the $F(h, \tau)=0$ has only one positive root denoted by $h_{+}$. Furthermore, $F(\omega, \tau)=0$ has a unique positive root given by $\omega=\sqrt{h_{+}}$.

Define $\alpha(\tau) \in[0,2 \pi)$ such that $\sin \alpha(\tau)$ and $\cos \alpha(\tau)$ are given by the right hand sides of (37a) and (37b), respectively, with $\alpha(\tau)$ given by (38a) and (38b).

And the relation between the argument $\alpha$ and $\omega \tau$ in (37a) and (37b) for $\tau>0$ must be

$$
\omega \tau=\alpha+2 n \pi, \quad n=0,1,2, \ldots
$$

Hence we can define the maps $\tau_{n}: I \rightarrow R_{+0}$ given by

$$
\tau_{n}(\tau):=\frac{\alpha(\tau)+2 n \pi}{\omega(\tau)}, \quad \tau_{n}>0, n=0,1,2, \ldots,
$$

where a positive root $\omega(\tau)$ of $(42)$ exists in $I$.

Let one introduces the functions $S_{n}(\tau): I \rightarrow R$,

$$
S_{n}(\tau)=\tau-\frac{\alpha(\tau)+2 n \pi}{\omega(\tau)}, \quad n=0,1,2, \ldots,
$$

that are continuous and differentiable in $\tau$. Thus, we give the following theorem which is due to Beretta and Kuang [16].

Theorem 7. Assume that $\omega(\tau)$ is a positive root of (19) defined for $\tau \in I, I \subseteq R_{+0}$, and at some $\tau^{*} \in I, S_{n}\left(\tau^{*}\right)=0$ for some $n \in N_{0}$, then a pair of simple conjugate pure imaginary roots $\lambda= \pm i \omega$ exists at $\tau=\tau^{*}$ which crosses the imaginary axis from left to right if $\delta\left(\tau^{*}\right)>0$ and crosses the imaginary axis from right to left if $\delta\left(\tau^{*}\right)<0$, where

$$
\delta\left(\tau^{*}\right)=\operatorname{sign}\left\{F_{\omega}^{\prime}\left(\omega \tau^{*}, \tau^{*}\right)\right\} \operatorname{sign}\left\{\left.\frac{d S_{n}(\tau)}{d \tau}\right|_{\tau=\tau^{*}}\right\} .
$$

Applying Theorem 3 and the Hopf bifurcation theorem for functional differential equation [12], we can conclude the existence of a Hopf bifurcation as stated in the following theorem.

Theorem 8. For system (2), the following conclusions are hold.

(i) If $a_{1}(0)>0$ and the function $S_{0}(\tau)$ has no positive zero in $I$, then the equilibrium $E^{*}$ is asymptotically stable for all $\tau \in\left[0, \tau_{\max }\right)$.

(ii) If $a_{1}(0)>0$ and the function $S_{0}(\tau)$ has positive zero in $I$, then there exists $\tau^{*} \in I$, such that the equilibrium $E^{*}$ is asymptotically stable for $\tau \in\left[0, \tau^{*}\right)$ and becomes unstable for $\tau \in\left(\tau^{*}, \tau_{\max }\right)$, with a Hopf bifurcation occurring when $\tau=\tau^{*}$.

(iii) If $a_{1}(0)<0$ and the function $S_{n}(\tau)$ has no positive zeros in $I$, then the equilibrium $E^{*}$ is always unstable for all $\tau \in\left[0, \tau_{\max }\right)$.

(iv) If $a_{1}(0)<0$ and the function $S_{n}(\tau)$ has positive zeros in $I$, for some $n \in N$, then there exists $\tau^{*} \in I$, such that the equilibrium $E^{*}$ is unstable for $0 \leq \tau<\tau^{*}$ and becomes asymptotically stable for $\tau \in\left(\tau^{*}, \tau_{\max }\right)$, with a Hopf bifurcation occurring when $\tau=\tau^{*}$.

Remark 9. If $\tau \geq(\ln \beta-\ln a(1+1 / K \varepsilon)) / m:=\tau_{\max }$, then $R_{0} \leq$ $1, y^{*} \leq 0$ and the equilibrium $E^{*}$ converges to $E_{1}=(K, 0)$.

\section{Direction and Stability of the Hopf Bifurcation of the DDEs}

In the above section, we have obtained some conditions which guarantee that the delayed predator-prey system with $\theta$-logistic growth and prey refuge undergoes the Hopf bifurcation at some value of $\tau=\tau^{*}$. In this section, we will study the direction, stability, and the period of the bifurcating 
periodic solutions. The approach we used here is based on the normal form approach and the center manifold theory introduced by Hassard et al. [17]. Throughout this section, we always assume that system (2) undergoes Hopf bifurcation at the positive equilibrium $E^{*}=\left(x^{*}, y^{*}\right)$ for $\tau=\tau^{*}$, and then $\pm i \omega$ is corresponding purely imaginary roots of the characteristic equation at the positive equilibrium $E^{*}=$ $\left(x^{*}, y^{*}\right)$.

Let $u_{1}(t)=x(t)-x^{*}, u_{2}(t)=y(t)-y^{*}$.

System (2) is transformed into

$$
\begin{aligned}
\dot{u}_{1}(t)= & \alpha_{1} u_{1}(t)+\alpha_{2} u_{2}(t)+\sum_{i+j \geq 2} \frac{1}{i ! j !} f_{i j}^{(1)} u_{1}^{i}(t) u_{2}^{j}(t), \\
\dot{u}_{2}(t)= & \beta_{1} u_{2}(t)+\beta_{2} u_{1}(t-\tau)+\beta_{3} u_{2}(t-\tau) \\
& +\sum_{i+j+l \geq 2} \frac{1}{i ! j ! l !} f_{i j l}^{(2)} u_{2}^{i}(t) u_{1}^{j}(t-\tau) u_{2}^{l}(t-\tau),
\end{aligned}
$$

where

$$
\begin{gathered}
\alpha_{1}=-r \theta\left(\frac{x^{*}}{K}\right)^{\theta}+\frac{\beta \varepsilon^{2} y^{*}}{\left(1+\varepsilon x^{*}\right)^{2}}, \\
\alpha_{2}=-\frac{\beta \varepsilon x^{*}}{1+\varepsilon x^{*}}, \\
\beta_{1}=-a, \\
\beta_{2}=\frac{\beta \varepsilon y^{*} e^{-m \tau}}{\left(1+\varepsilon x^{*}\right)^{2}}, \\
\beta_{3}=\frac{\beta \varepsilon x^{*} e^{-m \tau}}{1+\varepsilon x^{*}}, \\
f_{i j l}^{(2)}=\left.\frac{f^{(1)}=r x\left[1-\left(\frac{x}{K}\right)^{\theta}\right]-\frac{\beta \varepsilon x y}{1+\varepsilon x}}{\partial y^{i} \partial x(t-\tau)^{j} \partial y(t-\tau)^{l}}\right|_{\left(y^{*}, x^{*}, y^{*}\right)}, \quad i, j, l \geq 0 . \\
f^{(2)}=\frac{e^{-m \tau} \beta \varepsilon x(t-\tau) y(t-\tau)}{1+\varepsilon x(t-\tau)}-a y, \\
f_{i j}^{(1)}=\left.\frac{\partial^{i+j} f^{(1)}}{\partial x^{i} \partial y^{j}}\right|_{\left(x^{*}, y^{*}\right)} ^{(2)},
\end{gathered}
$$

For the simplicity of notations, we rewrite (50) as

$$
\dot{u}(t)=L_{v}\left(u_{t}\right)+f\left(v, u_{t}\right),
$$

where $u(t)=\left(u_{1}(t), u_{2}(t)\right)^{T} \in R^{2}, u_{t}(\zeta) \in C$ is defined by $u_{t}(\zeta)=u(t+\zeta)$, and $L_{v}: C \rightarrow R^{2}, f: R \times C \rightarrow R^{2}$ are given, respectively, by

$$
\begin{gathered}
L_{v}(\phi)=\left(\begin{array}{cc}
\alpha_{1} & \alpha_{2} \\
0 & \beta_{1}
\end{array}\right) \phi(0)+\left(\begin{array}{cc}
0 & 0 \\
\beta_{2} & \beta_{3}
\end{array}\right) \phi(-\tau), \\
f(v, \phi)=\left(\begin{array}{c}
\sum_{i+j \geq 2} \frac{1}{i ! j !} f_{i j}^{(1)} \phi_{1}^{i}(t) \phi_{2}^{j}(t) \\
\sum_{i+j+l \geq 2} \frac{1}{i ! j ! l !} f_{i j l}^{(2)} \phi_{2}^{i}(t) \phi_{1}^{j}(t-\tau) \phi_{2}^{l}(t-\tau)
\end{array}\right) .
\end{gathered}
$$

By the Riesz representation theorem, there exists a function $\eta(\zeta, v)$ of bounded variation for $\zeta \in[-\tau, 0]$, such that

$$
L_{v}(\phi)=\int_{-\tau}^{0} d \eta(\zeta, 0) \phi(\zeta) \quad \text { for } \phi \in C .
$$

In fact, we can choose

$$
\eta(\zeta, v)=\left(\begin{array}{cc}
\alpha_{1} & \alpha_{2} \\
0 & \beta_{1}
\end{array}\right) \delta(\zeta)-\left(\begin{array}{cc}
0 & 0 \\
\beta_{2} & \beta_{3}
\end{array}\right) \delta(\zeta+\tau),
$$

where $\delta$ denotes the Dirac delta function. For $\phi \in$ $C^{1}\left([-\tau, 0], R^{2}\right)$, define

$$
\begin{gathered}
A(v) \phi= \begin{cases}\frac{d \phi(\zeta)}{d \zeta}, & \zeta \in[-\tau, 0), \\
\int_{-\tau}^{0} d \eta(s, v) \phi(s), & \zeta=0,\end{cases} \\
R(v) \phi= \begin{cases}0, & \zeta \in[-\tau, 0), \\
f(v, \phi), & \zeta=0 .\end{cases}
\end{gathered}
$$

Then system (52) is equivalent to

$$
\dot{u}(t)=A(v) u_{t}+R(v) u_{t},
$$

where $u_{t}(\zeta)=u(t+\zeta)$ for $\zeta \in[-\tau, 0]$.

For $\psi \in C^{1}\left([0, \tau],\left(R^{2}\right)^{*}\right)$, define

$$
A^{*} \psi(s)= \begin{cases}-\frac{d \psi(s)}{d s}, & s \in(0, \tau], \\ \int_{-\tau}^{0} \psi(-t) d \eta(t, 0), & s=0,\end{cases}
$$

and a bilinear inner product

$$
\begin{aligned}
\langle\psi(s), \phi(\zeta)\rangle= & \bar{\psi}(0) \phi(0) \\
& -\int_{-\tau}^{0} \int_{\xi=0}^{\zeta} \bar{\psi}(\xi-\zeta) d \eta(\zeta) \phi(\xi) d \xi,
\end{aligned}
$$

where $\eta(\zeta)=\eta(\zeta, 0)$. Then $A(0)$ and $A^{*}$ are adjoint operators. By the discussion in Section 4 , we know that $\pm i \omega^{*} \tau^{*}$ are eigenvalues of $A(0)$. Thus, they are also eigenvalues of $A^{*}$. We first need to compute the eigenvector of $A(0)$ and $A^{*}$ corresponding to $i \omega^{*} \tau^{*}$ and $-i \omega^{*} \tau^{*}$, respectively.

Suppose that $q(\zeta)=(1, q)^{T} e^{i \omega^{*} \tau^{*} \zeta}$ is the eigenvector of $A(0)$ corresponding to $i \omega^{*} \tau^{*}$; then $A(0) q(\zeta)=i \omega^{*} \tau^{*} q(\zeta)$. It follows from (55) and (56) and the definition of $A(0)$ that

$$
\left(\begin{array}{cc}
\alpha_{1}-i \omega^{*} & \alpha_{2} \\
\beta_{2} e^{-i \omega^{*} \tau^{*}} & \beta_{1}+\beta_{3} e^{-i \omega^{*} \tau^{*}}-i \omega^{*}
\end{array}\right) q(0)=\left(\begin{array}{l}
0 \\
0
\end{array}\right) .
$$


Solving the equations above, we derive that

$$
q(0)=(1, \rho)^{T}=\left(1, \frac{i \omega^{*}-\alpha_{1}}{\alpha_{2}}\right)^{T} .
$$

On the other hand, suppose that $q^{*}(s)=D(1, \sigma) e^{i \omega^{*} \tau^{*} s}$ is the eigenvector of $A^{*}$ corresponding to $-i \omega^{*} \tau^{*}$. It follows from (55) and (56) and the definition of $A^{*}$ that

$$
\left(\begin{array}{cc}
\alpha_{1}+i \omega^{*} & \beta_{2} e^{i \omega^{*} \tau^{*}} \\
\alpha_{2} & \beta_{1}+\beta_{3} e^{i \omega^{*} \tau^{*}}+i \omega^{*}
\end{array}\right)\left(q^{*}(0)\right)^{T}=\left(\begin{array}{l}
0 \\
0
\end{array}\right),
$$

which yields

$$
q^{*}(0)=D(1, \sigma)=D\left(1,-\frac{\alpha_{1}+i \omega^{*}}{\beta_{2}} e^{-i \omega^{*} \tau^{*}}\right) .
$$

In order to assure $\left\langle q^{*}(s), q(\zeta)\right\rangle=1$, we need to determine the value of $D$. By (58), we have

$$
\begin{aligned}
& \left\langle q^{*}(s), q(\zeta)\right\rangle \\
& =\bar{D}(1, \bar{\sigma})(1, \rho)^{T} \\
& \quad-\int_{-\tau^{*}}^{0} \int_{\xi=0}^{\zeta} \bar{D}(1, \bar{\sigma}) e^{-i \omega^{*} \tau^{*}(\xi-\zeta)} d \eta(\zeta)(1, \rho)^{T} e^{i \omega^{*} \tau^{*} \xi} d \xi \\
& =\bar{D}\left\{1+\rho \bar{\sigma}-\int_{-\tau^{*}}^{0}(1, \bar{\sigma}) e^{i \omega^{*} \tau^{*} \zeta} d \eta(\zeta)(1, \rho)^{T}\right\} \\
& =\bar{D}\left\{1+\rho \bar{\sigma}+\left(\beta_{2}+\rho \beta_{3}\right) \bar{\sigma} \tau^{*} e^{i \omega^{*} \tau^{*}}\right\} .
\end{aligned}
$$

Thus, we can choose $D$ as

$$
D=\frac{1}{1+\rho \bar{\sigma}+\left(\beta_{2}+\rho \beta_{3}\right) \bar{\sigma} \tau^{*} e^{i \omega^{*} \tau^{*}}}
$$

such that

$$
\left\langle q^{*}(s), q(\zeta)\right\rangle=1, \quad\left\langle q^{*}(s), \bar{q}(\zeta)\right\rangle=0 .
$$

In the following, we apply the ideas in Hassard et al. [17] to describe the center manifold $C_{0}$ at $\mu=0$, similar to that in $[18,19]$. Let $u_{t}$ be the solution of (52) when $\mu=0$. Define

$$
z(t)=\left\langle q^{*}, u_{t}\right\rangle, \quad W(t, \zeta)=u_{t}(\zeta)-2 \operatorname{Re}\{z(t) q(\zeta)\} .
$$

On the center manifold $C_{0}$, we have

$$
W(t, \zeta)=W(z(t), \bar{z}(t), \zeta)
$$

where

$$
\begin{aligned}
& W(z(t), \bar{z}(t), \zeta) \\
& \quad=W_{20}(\zeta) \frac{z^{2}}{2}+W_{11}(\zeta) z \bar{z}+W_{02}(\zeta) \frac{\bar{z}^{2}}{2}+\cdots
\end{aligned}
$$

and $z$ and $\bar{z}$ are local coordinates for center manifold $C_{0}$ in the direction of $q^{*}$ and $\bar{q}^{*}$. Note that $W$ is real if $u_{t}$ is real.
We only consider real solutions. For solution $u_{t} \in C_{0}$ of (52), since $\mu=0$, we have

$$
\begin{aligned}
\dot{z}(t) & =i \omega^{*} \tau^{*} z+\bar{q}^{*}(0) f(0, W(z, \bar{z}, 0)+2 \operatorname{Re}\{z q(\zeta)\}) \\
& =i \omega^{*} \tau^{*} z+\bar{q}^{*}(0) f_{0}(z, \bar{z}) .
\end{aligned}
$$

We rewrite this equation as

$$
\dot{z}(t)=i \omega^{*} \tau^{*} z+\mathrm{g}(z, \bar{z})
$$

where

$$
\begin{aligned}
g(z, \bar{z}) & =\bar{q}^{*}(0) f_{0}(z, \bar{z}) \\
& =g_{20} \frac{z^{2}}{2}+g_{11} z \bar{z}+g_{02} \frac{\bar{z}^{2}}{2}+g_{21} \frac{z^{2} \bar{z}}{2}+\cdots
\end{aligned}
$$

It follows from $(68)$ that $u_{t}(\zeta)=W(t, \zeta)+z q(\zeta)+\bar{z} \bar{q}(\zeta)$ and $q(\zeta)=(1, \rho)^{T} e^{i \omega^{*} \tau^{*} \zeta}$, and then

$$
\begin{aligned}
u_{1 t}(0)= & z+\bar{z}+W_{20}^{(1)}(0) \frac{z^{2}}{2}+W_{11}^{(1)}(0) z \bar{z} \\
& +W_{02}^{(1)}(0) \frac{\bar{z}^{2}}{2}+\cdots,
\end{aligned}
$$

$$
\begin{aligned}
u_{1 t}\left(-\tau^{*}\right)= & z e^{-i \omega^{*} \tau^{*}}+\bar{z} e^{i \omega^{*} \tau^{*}}+W_{20}^{(1)}\left(-\tau^{*}\right) \frac{z^{2}}{2} \\
& +W_{11}^{(1)}\left(-\tau^{*}\right) z \bar{z}+W_{02}^{(1)}\left(-\tau^{*}\right) \frac{\bar{z}^{2}}{2}+\cdots,
\end{aligned}
$$$$
u_{2 t}(0)=z \rho+\bar{z} \bar{\rho}+W_{20}^{(2)}(0) \frac{z^{2}}{2}+W_{11}^{(2)}(0) z \bar{z}
$$

$$
+W_{02}^{(2)}(0) \frac{\bar{z}^{2}}{2}+\cdots,
$$

$$
\begin{aligned}
u_{2 t}\left(-\tau^{*}\right)= & z \rho e^{-i \omega^{*} \tau^{*}}+\bar{z} \bar{\rho} e^{i \omega^{*} \tau^{*}}+W_{20}^{(2)}\left(-\tau^{*}\right) \frac{z^{2}}{2} \\
& +W_{11}^{(2)}\left(-\tau^{*}\right) z \bar{z}+W_{02}^{(2)}\left(-\tau^{*}\right) \frac{\bar{z}^{2}}{2}+\cdots
\end{aligned}
$$

It follows from (54) and (73) that 
$g_{20}$

$$
\begin{aligned}
=\bar{D}\left[f_{20}^{(1)}\right. & +f_{02}^{(1)} \rho^{2}+2 f_{11}^{(1)} \rho \\
+ & \bar{\sigma}\left(2 f_{011}^{(2)} \rho e^{-2 i \omega^{*} \tau^{*}}+f_{020}^{(2)} e^{-2 i \omega^{*} \tau^{*}}\right. \\
& \left.\left.+f_{002}^{(2)} \rho^{2} e^{-2 i \omega^{*} \tau^{*}}\right)\right],
\end{aligned}
$$

$g_{11}$

$$
\begin{aligned}
=\bar{D}[ & f_{20}^{(1)}+f_{02}^{(1)} \rho \bar{\rho}+f_{11}^{(1)}(\rho+\bar{\rho}) \\
& \left.+\bar{\sigma}\left(f_{011}^{(2)}(\rho+\bar{\rho})+f_{020}^{(2)}+f_{002}^{(2)} \rho \bar{\rho}\right)\right],
\end{aligned}
$$

$g_{02}$

$$
\begin{gathered}
=\bar{D}\left[f_{20}^{(1)}+f_{02}^{(1)} \bar{\rho}^{2}+2 f_{11}^{(1)} \bar{\rho}\right. \\
+\bar{\sigma}\left(2 f_{011}^{(2)} \bar{\rho} e^{2 i \omega^{*} \tau^{*}}+f_{020}^{(2)} e^{2 i \omega^{*} \tau^{*}}\right. \\
\left.\left.+f_{002}^{(2)} \bar{\rho}^{2} e^{2 i \omega^{*} \tau^{*}}\right)\right],
\end{gathered}
$$

$g_{21}$

$=2 \bar{D}$

$\times\{\bar{\sigma}$

$\times\left[f_{011}^{(2)}\right.$

$$
\times\left(\frac{1}{2} e^{i \omega^{*} \tau^{*}} W_{20}^{(2)}\left(-\tau^{*}\right)+e^{-i \omega^{*} \tau^{*}} W_{11}^{(2)}\left(-\tau^{*}\right)\right.
$$$$
\left.+\rho e^{-i \omega^{*} \tau^{*}} W_{11}^{(1)}\left(-\tau^{*}\right)+\frac{1}{2} \bar{\rho} e^{i \omega^{*} \tau^{*}} W_{20}^{(1)}\left(-\tau^{*}\right)\right)
$$$$
+f_{020}^{(2)}\left(e^{-i \omega^{*} \tau^{*}} W_{11}^{(1)}\left(-\tau^{*}\right)+\frac{1}{2} e^{i \omega^{*} \tau^{*}} W_{20}^{(1)}\left(-\tau^{*}\right)\right)
$$$$
+f_{002}^{(2)}\left(\rho e^{-i \omega^{*} \tau^{*}} W_{11}^{(2)}\left(-\tau^{*}\right)+\frac{1}{2} e^{i \omega^{*} \tau^{*}} W_{20}^{(2)}\left(-\tau^{*}\right)\right)
$$$$
+\frac{1}{2} f_{030}^{(2)} e^{-i \omega^{*} \tau^{*}}+\frac{1}{2} f_{003}^{(2)} \rho^{2} \bar{\rho} e^{-i \omega^{*} \tau^{*}}
$$$$
\left.+\frac{1}{2} f_{012}^{(2)} \rho(\rho+2 \bar{\rho}) e^{-i \omega^{*} \tau^{*}}+\frac{1}{2} f_{021}^{(2)}(\rho+2 \bar{\rho}) e^{-i \omega^{*} \tau^{*}}\right]
$$$$
+\left[f_{20}^{(1)}\left(W_{11}^{(1)}(0)+\frac{1}{2} W_{20}^{(1)}(0)\right)\right.
$$$$
+f_{02}^{(1)}\left(\rho W_{11}^{(2)}(0)+\frac{1}{2} \bar{\rho} W_{20}^{(2)}(0)\right)
$$$$
+f_{11}^{(1)}\left(W_{11}^{(2)}(0)+\frac{1}{2} W_{20}^{(2)}(0)\right.
$$

$$
\left.\left.\left.+\rho W_{11}^{(1)}(0)+\frac{1}{2} \rho W_{20}^{(1)}(0)\right)\right]\right\} .
$$

We now calculate $W_{20}(\zeta)$ and $W_{11}(\zeta)$. It follows from (58) and (68) that

$$
\begin{aligned}
\dot{W} & =\dot{u}_{t}-\dot{z} q-\dot{\bar{z}} \bar{q} \\
& = \begin{cases}A(0) W-2 \operatorname{Re}\left\{\bar{q}^{*} f_{0} q(\zeta)\right\}, & s \in\left[-\tau^{*}, 0\right), \\
A(0) W-2 \operatorname{Re}\left\{\bar{q}^{*} f_{0} q(0)\right\}+f_{0}, & \zeta=0,\end{cases} \\
& :=A W+H(z, \bar{z}, \zeta),
\end{aligned}
$$

where

$$
H(z, \bar{z}, \zeta)=H_{20}(\zeta) \frac{z^{2}}{2}+H_{11}(\zeta) z \bar{z}+H_{02}(\zeta) \frac{\bar{z}^{2}}{2}+\cdots
$$

On the other hand, on $C_{0}$ near the origin

$$
\dot{W}=W_{z} \dot{z}+W_{\bar{z}} \dot{\bar{z}}
$$

We derive from (76)-(78) that

$$
\left(A-2 i \omega_{0}\right) W_{20}(\zeta)=-H_{20}(\zeta), \quad A W_{11}(\zeta)=-H_{11}(\zeta), \ldots
$$

It follows from (73) and (76) that for $\zeta \in\left[-\tau^{*}, 0\right)$,

$$
H(z, \bar{z}, \zeta)=-\bar{q}^{*} f_{0} q(\zeta)-q^{*} \bar{f}_{0} \bar{q}(\zeta)=-\mathrm{g} q(\zeta)-\bar{g} \bar{q}(\zeta) .
$$

Comparing the coefficients in (77) gives that for $\zeta \in\left[-\tau^{*}, 0\right)$,

$$
\begin{aligned}
& H_{20}(\zeta)=-g_{20} q(\zeta)-\bar{g}_{02} \bar{q}(\zeta), \\
& H_{11}(\zeta)=-g_{11} q(\zeta)-\bar{g}_{11} \bar{q}(\zeta) .
\end{aligned}
$$

From (79), (81), and following the definition of $A$, we get

$$
\dot{W}_{20}=2 i \omega^{*} \tau^{*} W_{20}(\zeta)+g_{20} q(\zeta)+\bar{g}_{20} \bar{q}(\zeta) .
$$

Notice that $q(\zeta)=q(0) e^{i \omega^{*} \tau^{*} \zeta}$, and hence

$$
W_{20}=\frac{i g_{20}}{\omega^{*} \tau^{*}} q(0) e^{i \omega^{*} \tau^{*} \zeta}+\frac{i \bar{g}_{02}}{\omega^{*} \tau^{*}} \bar{q}(0) e^{-i \omega^{*} \tau^{*} \zeta}+E_{1} e^{2 i \omega^{*} \tau^{*} \zeta},
$$

where $E_{1}=\left(E_{1}^{1}, E_{1}^{2}\right) \in R^{2}$ is a constant vector. Similarly, from (79) and (82), we obtain

$$
W_{11}=-\frac{i g_{11}}{\omega^{*} \tau^{*}} q(0) e^{i \omega^{*} \tau^{*} \zeta}+\frac{i \bar{g}_{11}}{\omega^{*} \tau^{*}} \bar{q}(0) e^{-i \omega^{*} \tau^{*} \zeta}+E_{2},
$$

where $E_{2}=\left(E_{2}^{1}, E_{2}^{2}\right) \in R^{2}$ is also a constant vector.

In what follows, we will seek appropriate $E_{1}$ and $E_{2}$. From the definition of $A$ and (79), we obtain that

$$
\begin{gathered}
\int_{-\tau^{*}}^{0} d \eta(\zeta) W_{20}(\zeta)=2 i \omega_{0}-H_{20}(0), \\
\int_{-\tau^{*}}^{0} d \eta(\zeta) W_{11}(\zeta)=-H_{11}(0),
\end{gathered}
$$




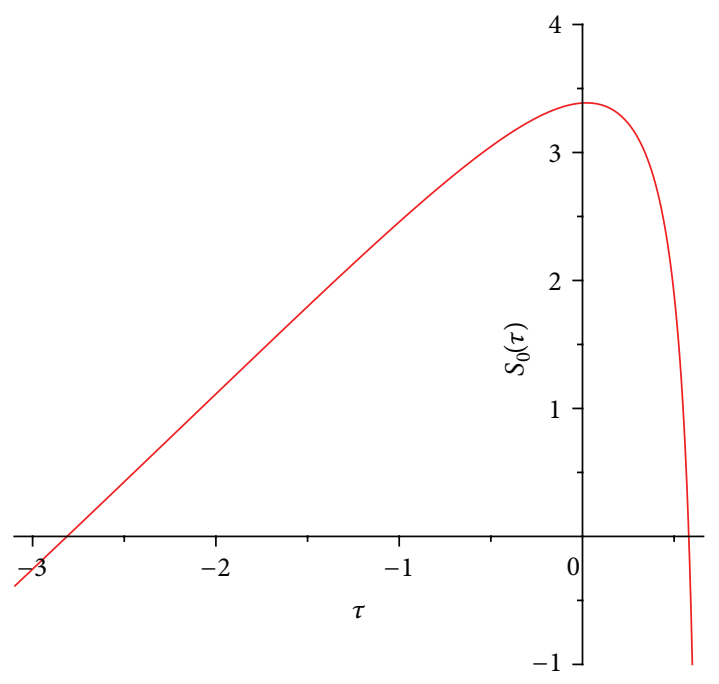

FIGURE 3: Graph of the function $S_{0}(\tau)$.

where $\eta(\zeta)=\eta(0, \zeta)$.

Set

$$
\begin{aligned}
& d_{1}=f_{20}^{(1)}+f_{02}^{(1)} \rho^{2}+2 f_{11}^{(1)} \rho, \\
& d_{2}=2 f_{011}^{(2)} \rho e^{-2 i \omega^{*} \tau^{*}}+f_{020}^{(2)} e^{-2 i \omega^{*} \tau^{*}}+f_{002}^{(2)} \rho^{2} e^{-2 i \omega^{*} \tau^{*}}, \\
& d_{3}=f_{20}^{(1)}+f_{02}^{(1)} \rho \bar{\rho}+f_{11}^{(1)}(\rho+\bar{\rho}), \\
& d_{4}=f_{011}^{(2)}(\rho+\bar{\rho})+f_{020}^{(2)}+f_{002}^{(2)} \rho \bar{\rho} .
\end{aligned}
$$

By (76), we have

$$
\begin{aligned}
& H_{20}(\zeta)=-g_{20} q(0)-\bar{g}_{20} \bar{q}(0)+\left[\begin{array}{l}
d_{1} \\
d_{2}
\end{array}\right], \\
& H_{11}(\zeta)=-g_{11} q(0)-\bar{g}_{11} \bar{q}(0)+\left[\begin{array}{l}
d_{3} \\
d_{4}
\end{array}\right] .
\end{aligned}
$$

Substituting (84) and (89) into (86), we obtain

$$
\left(2 i \omega^{*} \tau^{*} I-\int_{-\tau^{*}}^{0} e^{2 i \omega^{*} \tau^{*} \zeta} d \eta(\zeta)\right) E_{1}=\left[\begin{array}{l}
d_{1} \\
d_{2}
\end{array}\right],
$$

which leads to

$$
A E_{1}=\left[\begin{array}{l}
d_{1} \\
d_{2}
\end{array}\right]
$$

where

$$
A=\left[\begin{array}{cc}
2 i \omega^{*} \tau^{*}-\alpha_{1} & -\alpha_{2} \\
-\beta_{2} e^{-2 i \omega^{*} \tau^{*}} & 2 i \omega^{*} \tau^{*}-\beta_{1}-\beta_{3} e^{-2 i \omega^{*} \tau^{*}}
\end{array}\right] .
$$

It follows that

$$
\begin{gathered}
E_{1}^{(1)}=\frac{1}{|A|}\left|\begin{array}{cc}
d_{1} & -\alpha_{2} \\
d_{2} & 2 i \omega^{*} \tau^{*}-\beta_{1}-\beta_{3} e^{-2 i \omega^{*} \tau^{*}}
\end{array}\right|, \\
E_{1}^{(2)}=\frac{1}{|A|}\left|\begin{array}{cc}
2 i \omega^{*} \tau^{*}-\alpha_{1} & d_{1} \\
-\beta_{2} e^{-2 i \omega^{*} \tau^{*}} & d_{2}
\end{array}\right| .
\end{gathered}
$$

Similarly, substituting (85) and (90) into (87), we can get

$$
B E_{2}=\left[\begin{array}{l}
d_{3} \\
d_{4}
\end{array}\right]
$$

where

$$
B=\left[\begin{array}{cc}
-\alpha_{1} & -\alpha_{2} \\
-\beta_{2} & -\beta_{1}-\beta_{3}
\end{array}\right]
$$

and hence

$$
\begin{gathered}
E_{2}^{(1)}=\frac{1}{|B|}\left|\begin{array}{cc}
d_{3} & -\alpha_{2} \\
d_{4} & -\beta_{1}-\beta_{3}
\end{array}\right|, \\
E_{2}^{(2)}=\frac{1}{|B|}\left|\begin{array}{ll}
-\alpha_{1} & d_{3} \\
-\beta_{2} & d_{4}
\end{array}\right| .
\end{gathered}
$$

Thus, we can determine $W_{20}$ and $W_{11}$ from (84) and (85). Furthermore, $g_{21}$ in (75) can be expressed by the parameters and delay given in system (50). Based on the above analysis, we can see that each $g_{i j}$ can be determined by the parameters. Thus we can compute the following quantities:

$$
\begin{aligned}
c_{1}(0) & =\frac{i}{2 \omega^{*} \tau^{*}}\left(g_{11} g_{20}-2\left|g_{11}\right|^{2}-\frac{\left|g_{02}\right|^{2}}{3}\right)+\frac{g_{21}}{2}, \\
\mu_{2} & =-\frac{\operatorname{Re}\left\{c_{1}(0)\right\}}{\operatorname{Re}\left\{\lambda^{\prime}\left(\tau^{*}\right)\right\}}, \\
\beta_{2} & =2 \operatorname{Re}\left\{c_{1}(0)\right\}, \\
T_{2} & =-\frac{\operatorname{Im}\left\{c_{1}(0)\right\}+\mu_{2} \operatorname{Im}\left\{\lambda^{\prime}\left(\tau^{*}\right)\right\}}{\omega^{*} \tau^{*}} .
\end{aligned}
$$

Hence we have the following theorem by the result of Hassard et al. [17].

Theorem 10. In (98), $\mu_{2}$ determines the directions of the Hopf bifurcation: if $\mu_{2}>0\left(\mu_{2}<0\right)$, then the Hopf bifurcation is supercritical (subcritical) and the bifurcating periodic solutions exist for $\tau>\tau^{*}\left(\tau<\tau^{*}\right) ; \beta_{2}$ determines the stability of the bifurcating periodic solutions: the bifurcating periodic solutions are stable (unstable) if $\beta_{2}<0\left(\beta_{2}>0\right) ; T_{2}$ determines the period of the bifurcating periodic solutions: the period increases (decrease) if $\mathrm{T}_{2}>0(<0)$.

\section{Numerical Simulations and Discussion}

In this section, we will carry out some numerical simulations for supporting our theoretical analysis.

In the following, we choose two sets of parameters.

$$
\text { (i) } \begin{aligned}
r & =0.1, K=10, \beta=0.2, a=0.13, m=0.15, \theta=3, \\
\varepsilon & =0.42, \\
\text { (ii) } r & =0.1, K=10, \beta=0.2, a=0.13, m=0.15, \theta=3, \\
\varepsilon & =0.3 .
\end{aligned}
$$

For the parameters $(\mathrm{i}), \tau_{\max } \approx 1.45$ and $I=[0,1.45)$. The function $S_{0}(\tau)$ is drawn for $\tau \in I$ in Figure 3, from which we can see that there is only one positive critical value of the delay $\tau$, denoted by $\tau^{*}$, and $\tau^{*} \approx 0.58$. 


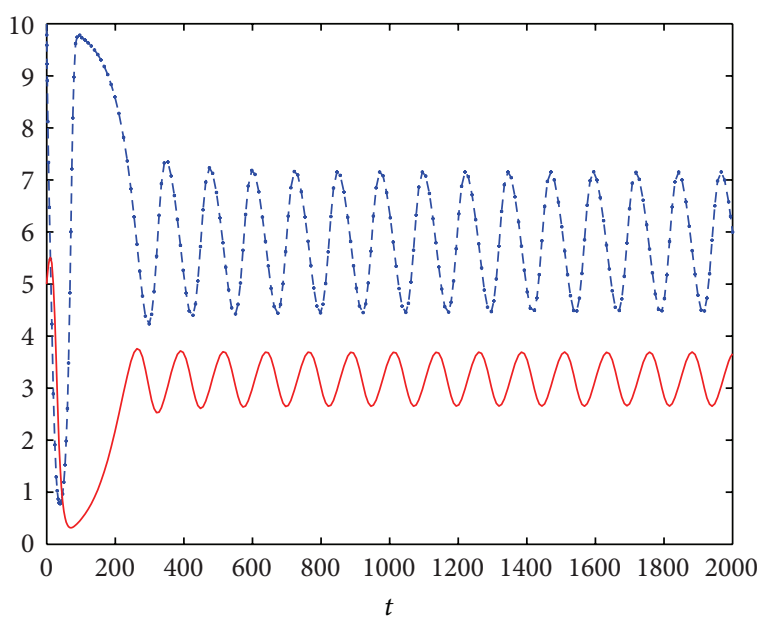

$-\cdots x$

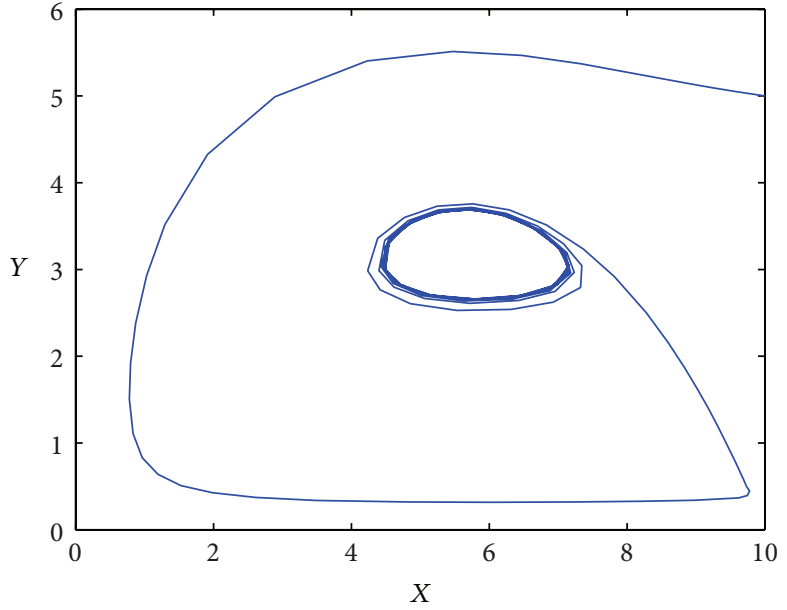

(b)

(a)

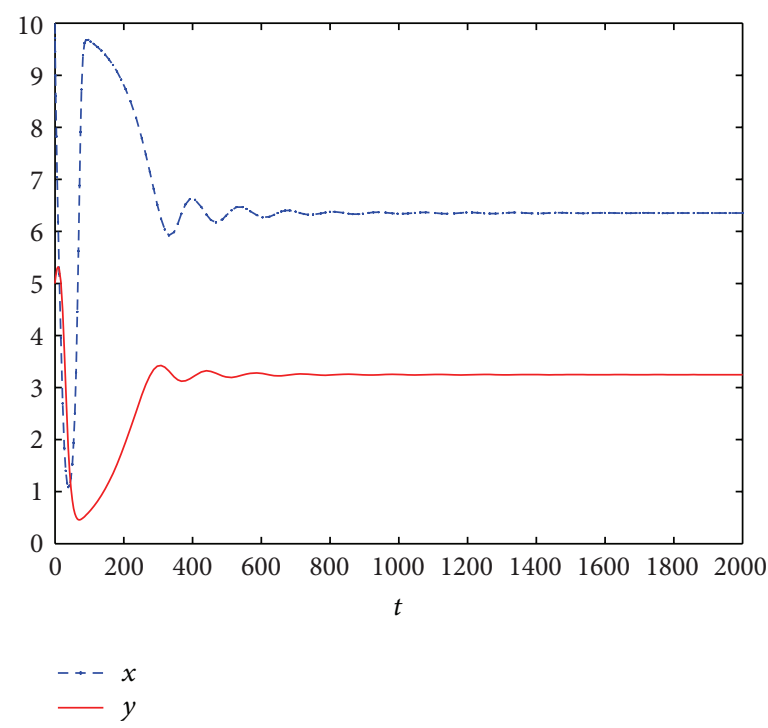

(c)

Figure 4: The infected steady state $E^{*}$ of system (2) is unstable when $\tau=0.55$ (a) (b) and stable when $\tau=0.75$ (c). The rest parameter values are given in (i), and the initial values are $x_{0}=8$ and $y_{0}=5$.

(1) When $\tau=0$, Figure 1(b) shows that the positive equilibrium of system (1) is unstable.

(2) When $\tau=0.55<\tau^{*}$, we can see the positive equilibrium of system (2) is unstable (see Figures 4(a) and $4(b))$.

(3) When $\tau=0.75 \in\left(\tau^{*}, \tau_{\max }\right)$, the positive equilibrium of system (2) is stable and there is a Hopf bifurcation that occurs when $\tau=\tau^{*}$. Furthermore, we can obtain $\operatorname{Re}\left(c_{1}(0)\right)<0$ and $\mu_{2}<0$. Therefore, the Hopf bifurcation of system (2) at the infected equilibrium is subcritical and the bifurcating periodic solutions are orbitally asymptotically stable (see Figure 4(b)).
(4) If $m=0$, that is the system (2) has no term $e^{-m \tau}$, then the positive equilibrium if it exists is always unstable for $\tau \geq 0$ (see Figures 1(b) and 5).

For the parameters (ii), the positive equilibrium of the DDEs is always stable for all $\tau \in I$ (see Figures 1(a) and 6).

In this paper, the Hopf bifurcation for a predator-prey system with $\theta$-logistic growth and prey refuge is studied. It is shown that the decreasing prey refuge rate or the increasing logistic index will derive the stable ODEs unstable. Therefore, the refuges used by prey have a stabilizing effect on the considered interactions [6-11].

For the DDEs, the time delay could be looked as a bifurcation parameter. Under certain condition, there is a critical delay $\tau^{*}$ and a $\tau_{\max }$, if the delay $\tau \in\left[0, \tau^{*}\right)$, the system 


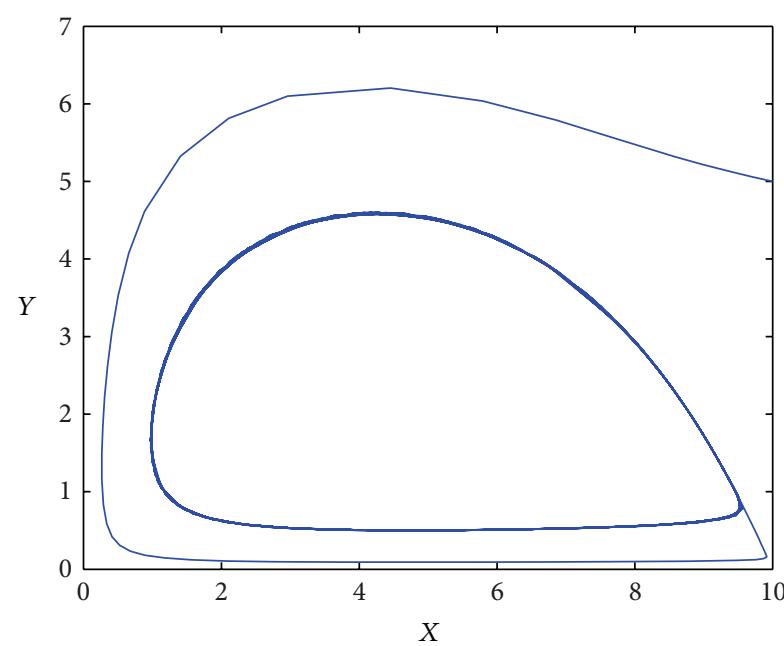

(a)

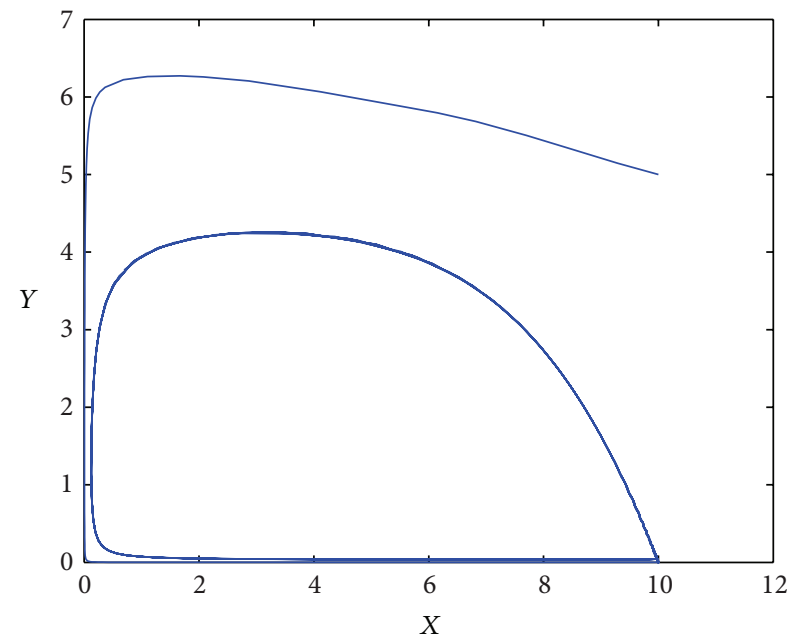

(b)

Figure 5: The infected steady state $E^{*}$ of system (2) without term $e^{-m \tau}$ is unstable when $\tau=0.75$ (a) and $\tau=10$ (b). The rest parameter values are given in (i), and the initial values are $x_{0}=8$ and $y_{0}=5$.

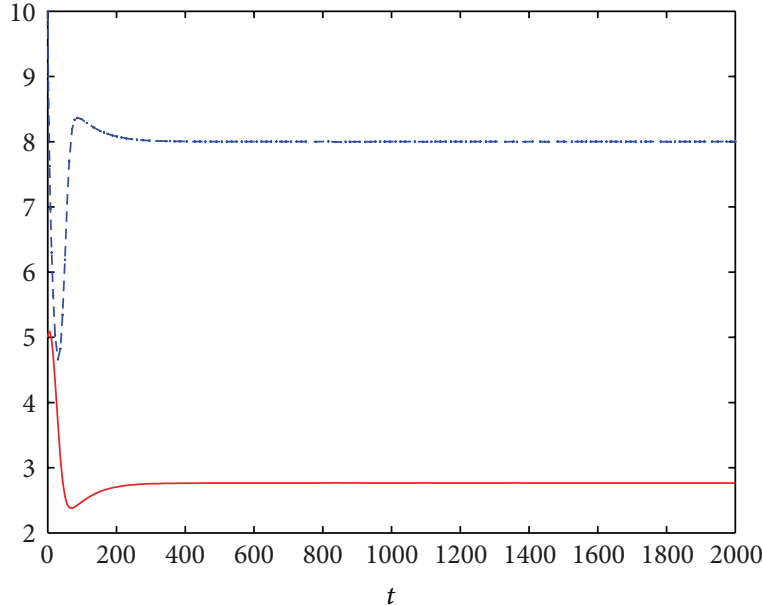

$$
-\cdots x
$$$$
-y
$$

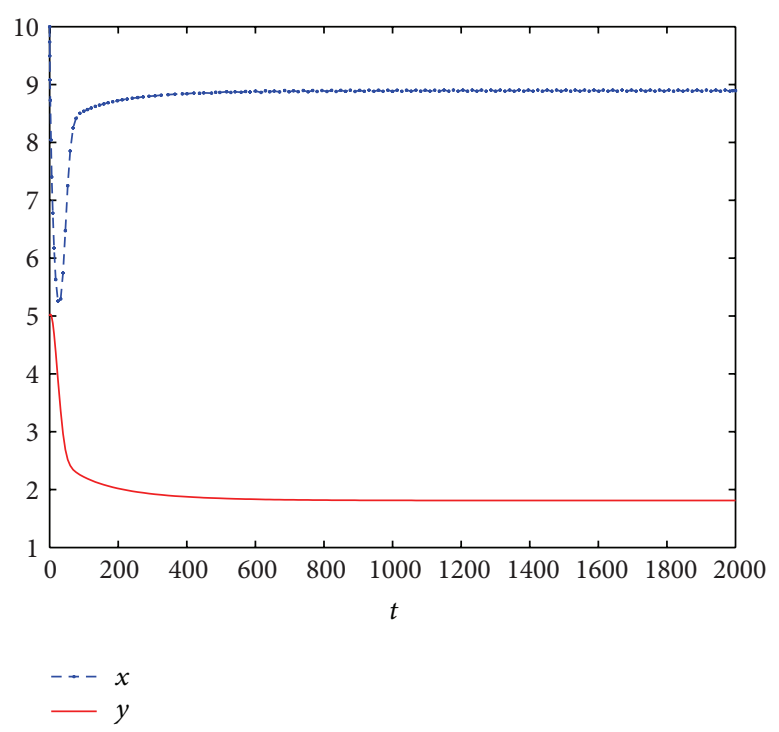

(b)

FIGURE 6: The infected steady state $E^{*}$ of system (2) is stable when $\tau=0.55$ (a) and $\tau=0.75$ (b). The rest parameter values are given in (ii), and the initial values are $x_{0}=8$ and $y_{0}=5$.

is unstable, and if $\tau \in\left(\tau^{*}, \tau_{\max }\right)$, the system is stable. But under this condition, the DDEs without the term $e^{-m \tau}$ will always be unstable.

\section{Acknowledgments}

The authors would like to thank the referees for their helpful suggestions, which improved the quality of this paper greatly. This work is supported by the National Natural Science Foundation of China (no. 11271298).

\section{References}

[1] C. S. Holling, "Some characteristics of simple types of predation and parasitism," Canadian Entomologist, vol. 91, pp. 385-398, 1959.

[2] M. P. Hassell and R. M. May, "Stability in insect host-parasite models," Journal of Animal Ecology, vol. 42, pp. 693-726, 1973.

[3] J. M. Smith, Models in Ecology, Cambridge University, Cambridge, UK, 1974.

[4] M. P. Hassell, The Dynamics of Arthropod Predator-Prey Systems, vol. 13, Princeton University, Princeton, NJ, USA, 1978. 
[5] L. Ji and C. Wu, "Qualitative analysis of a predator-prey model with constant-rate prey harvesting incorporating a constant prey refuge," Nonlinear Analysis: Real World Applications, vol. 11, no. 4, pp. 2285-2295, 2010.

[6] A. Sih, "Prey refuges and predator-prey stability," Theoretical Population Biology, vol. 31, no. 1, pp. 1-12, 1987.

[7] R. J. Taylor, Predation, Chapman and Hall, New York, NY, USA, 1984.

[8] E. González-Olivares and R. Ramos-Jiliberto, "Dynamic consequences of prey refuges in a simple model system: more prey, fewer predators and enhanced stability," Ecological Modelling, vol. 166, pp. 135-146, 2003.

[9] V. Krivan, "Effects of optimal antipredator behavior of prey on predator-prey dynamics: the role of refuges," Theoretical Population Biology, vol. 53, pp. 131-142, 1998.

[10] Z. Ma, W. Li, Y. Zhao, W. Wang, H. Zhang, and Z. Li, "Effects of prey refuges on a predator-prey model with a class of functional responses: the role of refuges," Mathematical Biosciences, vol. 218, no. 2, pp. 73-79, 2009.

[11] L. Chen, F. Chen, and L. Chen, "Qualitative analysis of a predator-prey model with Holling type II functional response incorporating a constant prey refuge," Nonlinear Analysis: Real World Applications, vol. 11, no. 1, pp. 246-252, 2010.

[12] Y. D. Tao, X. Wang, and X. Y. Song, "Effect of prey refuge on a harvested predator prey model with generalized functional response," Communications in Nonlinear Science and Numerical Simulation, vol. 16, pp. 1052-1059, 2010.

[13] W. Ko and K. Ryu, "A qualitative study on general Gause-type predator-prey models with constant diffusion rates," Journal of Mathematical Analysis and Applications, vol. 344, no. 1, pp. 217230, 2008.

[14] A. Tsoularis and J. Wallace, "Analysis of logistic growth models," Mathematical Biosciences, vol. 179, no. 1, pp. 21-55, 2002.

[15] J. Hale, Theory of Functional Differential Equations, Springer, Heidelberg, Germany, 1977.

[16] E. Beretta and Y. Kuang, "Geometric stability switch criteria in delay differential systems with delay dependent parameters," SIAM Journal on Mathematical Analysis, vol. 33, no. 5, pp. 11441165, 2002.

[17] B. D. Hassard, N. D. Kazarinoff, and Y. H. Wan, Theory and Applications of Hopf Bifurcation, vol. 41, Cambridge University Press, Cambridge, UK, 1981.

[18] Z. Ge and J. Yan, "Hopf bifurcation of a predator-prey system with stage structure and harvesting," Nonlinear Analysis: Theory, Methods \& Applications, vol. 74, no. 2, pp. 652-660, 2011.

[19] R. Xu, Q. Gan, and Z. Ma, "Stability and bifurcation analysis on a ratio-dependent predator-prey model with time delay," Journal of Computational and Applied Mathematics, vol. 230, no. 1, pp. 187-203, 2009. 


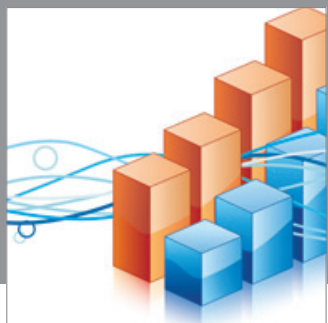

Advances in

Operations Research

mansans

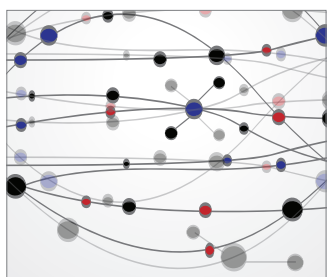

The Scientific World Journal
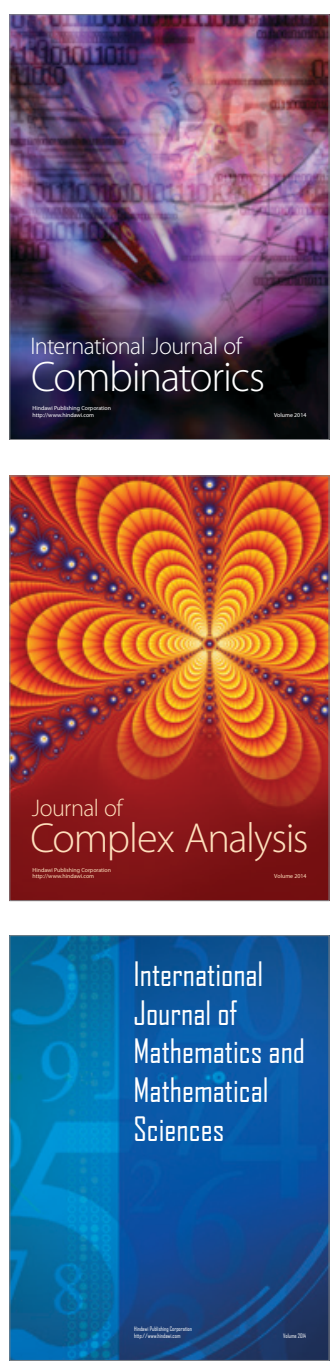
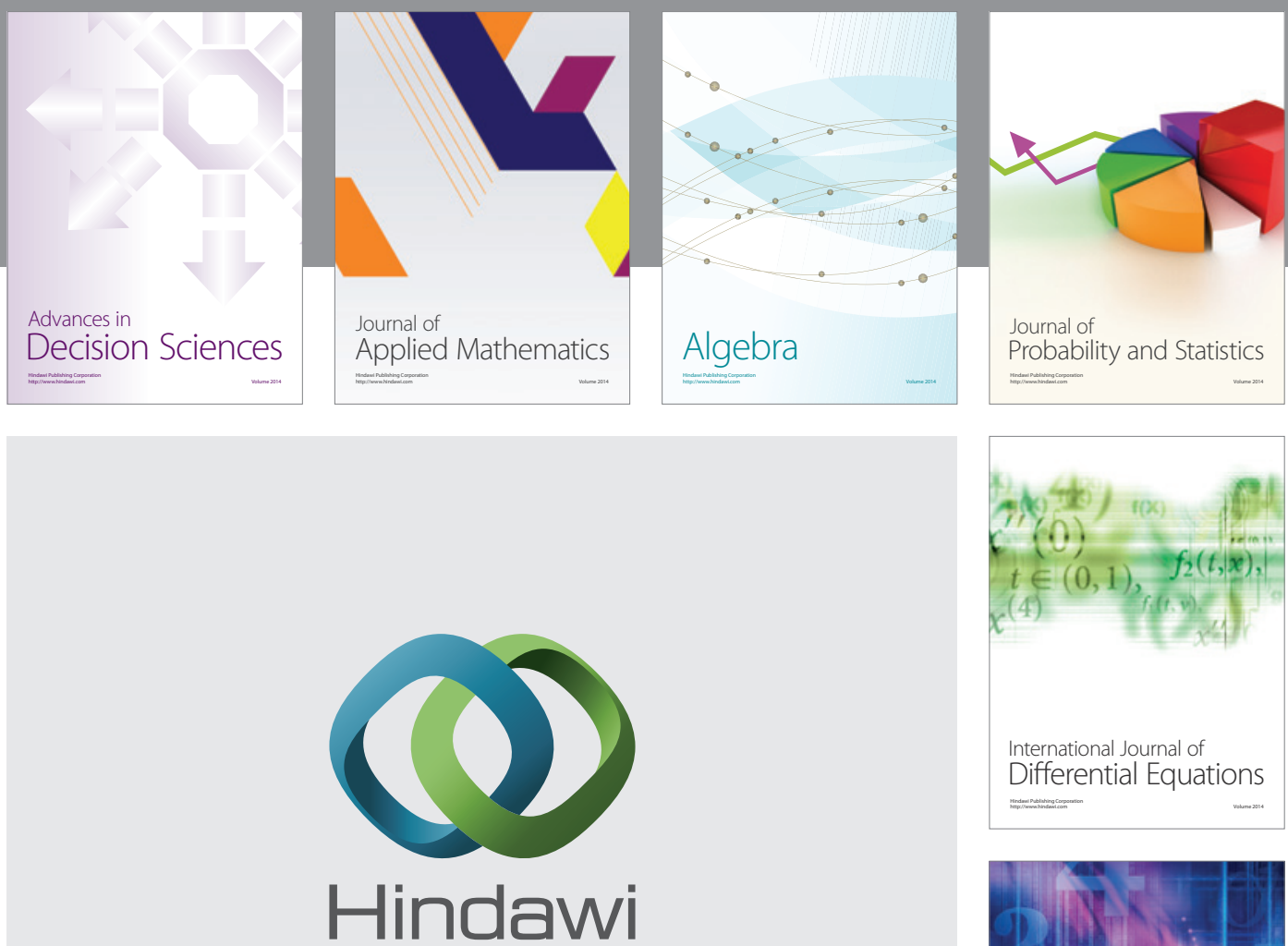

Submit your manuscripts at http://www.hindawi.com
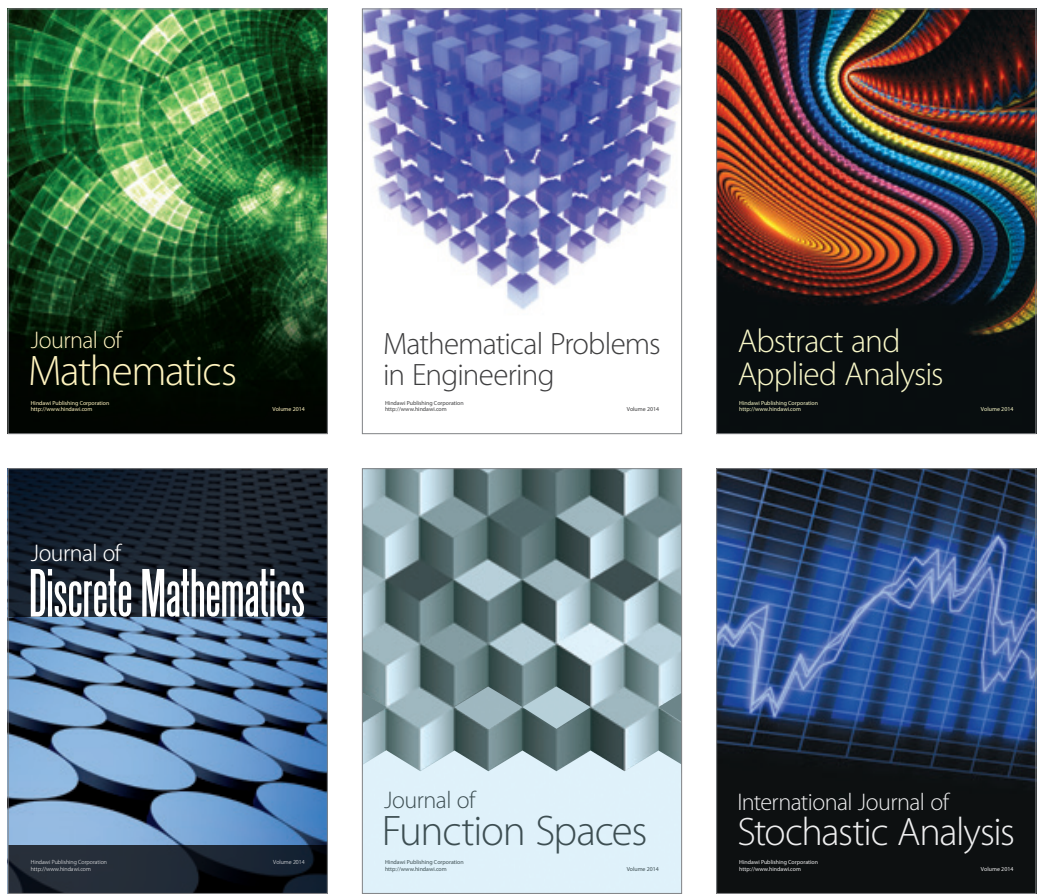

Journal of

Function Spaces

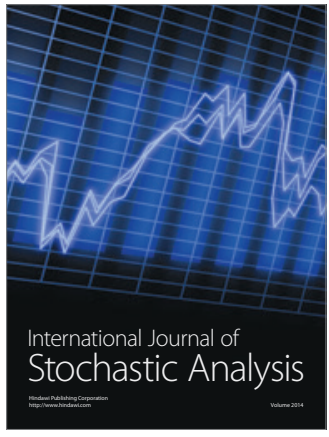

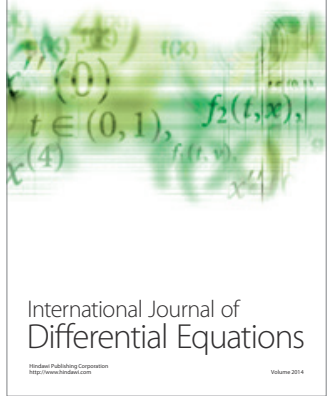
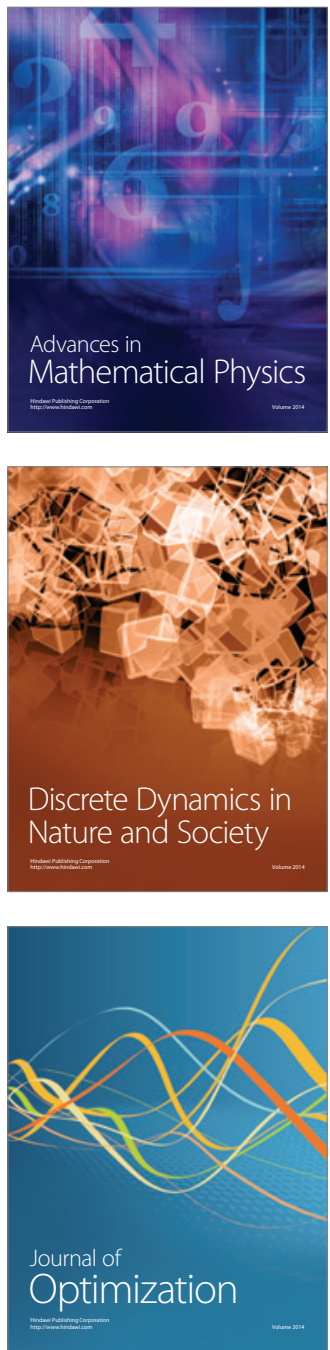